\title{
An approach for correcting magnitude and phase distortion in wideband piezoelectric transducer systems.
}

\author{
Said Assous, David Gunn, Claire Hopper, and Peter D Jackson \\ Ultra Sound Research Laboratory \\ British Geological Survey \\ Keyworth, NG12 5GG,UK \\ Email: sasso@bgs.ac.uk \\ Laurie Linnett \\ Fortkey Ltd, 1/18 Victoria Terrace \\ Edinburgh EH1 2JL,UK \\ Email: laurie@fortkey.com \\ Mike Lovell \\ Geology Department \\ University of Leicester, University Road \\ Leicester, LE1 7RH,UK
}

\begin{abstract}
Acoustic ultrasonic measurements are widespread and commonly performed using sensitive piezoelectric sensors. An accurate transducer system response to investigate pressure fluctuations in water and their subsequent detection remains a challenge. Typically, these sensors exploit the resonant behaviour of the piezoelectric active element, being designed to give maximum sensitivity in the bandwidth of interest. Calibration of such transducers can provide both magnitude and phase information describing the way in which the sensor responds to a surface displacement over its frequency range. Such resonant sensors are widely used for ultrasonic applications. The resonant nature of the sensors leads to the use of narrowband signals with central frequencies close to the resonant frequency of the piezoelectric element. Consequently, such devices work efficiently and linearly over only a narrow band of their overall frequency range. This causes phase and magnitude distortion of any linear broadband signal being transmitted through such a transmitter-receiver acoustic system. In the present work, we describe a software calibration technique to correct for distortion in a wideband piezoelectric transducer system. We consider only the input and the final output signals of the whole system. Compensating for the distortion of the magnitude and phase responses, we ensured the signal seen at the receiver represents a good replica of the desired signal. A Gaussian, linear, chirp signal was used to demonstrate our approach. This method may be applied to correct system distortion in a wide variety of ultrasonic applications.
\end{abstract}

Index Terms-Acoustic; Ultrasonic; Transducer; Calibration; Chirp Signal.

\section{INTRODUCTION}

Piezoelectric transducers are used both as transmitters and sensors in many ultrasonic applications such as non-destructive testing, underwater sonar, and medical imaging [1-4]. However, the transducer outputs are significantly affected by the coupling between the transducer and the other components (e.g. the amplifier, and medium in which the energy propagates) [5]. This means that coupling must be carefully considered if reliable acoustic signals are to be generated and used for system calibration. The overall accuracy measurement and bandwidth of the system is therefore limited by the performance of the transducers. A number of hardware techniques have been developed to design flat broadband frequency response matching networks [6-8] for acoustic transducers. The load is usually modelled as a resistor and capacitor [9] or as a simple four-element circuit [10]. The problem with this 
approach is that, typically, the frequency responses of piezoelectric elements have resonant characteristics, which are difficult to accurately mimic using fixed component networks. In most cases, improved results can be obtained if the network suggested by one of the techniques listed in refs [610] is used as a starting point for an optimization routine, which accounts for frequency dependent radiation resistance and reactance. Recently, Doust and Dix [11] introduced a hardware calibration technique, in which he demonstrated improved overall phase linearity, efficiency and amplitude response of transfer functions, in an electro-acoustic system. The calibration method of Doust and Dix seeks to improve the accuracy of wave shape measurements and transducer response. Specifically, it is a method and apparatus, which calibrates a system, comprising: amplifiers, filters, and analogto-digital converters. This is achieved by adding electronic equalisation devices between amplifier and transducer, removing phase and amplitude errors over a frequency spectrum. They called this technique equalisation. Distortion of the output signal in ultrasonic systems may be caused by many factors within the elements of the whole system, not only the transducer elements alone. It is often the case that the physical value (e.g. pressure) and the distorted waveform resulting from the conversion processes are repetitive with respect to time, current waveforms in alternating current power systems, for example. In these cases the original waveform of the physical value and the associated distorted waveform resulting from the conversion process can be decomposed into Fourier series of pure sine and cosine waves, with each wave having a unique frequency, amplitude and phase. At any frequency the transducer, amplifier, filter, or A/D converter can distort the signals introducing errors in the amplitude, or phase or both, which can in turn introduce distortion in reconstructed waveforms. These repetitive errors are frequency dependent; consequently, the hardware method and apparatus for digital calibration are incapable of correcting for them. A method and apparatus are needed for calibrating systems that convert time-varying physical values having repetitive waveforms, to accurate digital values. Doust's hardware calibration achieves this by taking into account all the subsystems and calibrating each subsystem in turn [12]. The software calibration method described below has the potential to remove amplitude and phase errors in the transducers, amplifiers, and analog- to-digital converters, by considering these devices as one system. Such a software calibration method has the benefit of simplicity and excludes a need to know the transfer function of each subsystem component (e.g. filter, amplifier, transducer). In essence, we consider the overall system as a 'black-box' and attempt to correct the output by compensating the input in terms of its phase and magnitude frequency responses.

\section{Methods}

This paper describes a software calibration method and associated procedures plus an experimental example, calibrating a system for its magnitude and phase response with respect to frequency; the experimental work was performed at the British Geological Survey Laboratories, Keyworth, UK. The need for calibration is highlighted when one realises the actual performance of a system may not be known and assumptions are being made as to the signal actually being injected into the medium. A prime purpose of this experiment is to show signals having precisely known amplitude and phase can be injected. Tone burst signals, used for the sensor calibration, were produced using a piezoelectric transducer driven by an Agilent 33120A function generator. The function generator produced tone-burst signals, which were digitised using a Tektronix TDS 3034B oscilloscope. To demonstrate this methodology of calibration, a series of measurements were performed using ultrasound transducers developed by Alba Ultrasound Ltd. These underwater transducers were designed to have a wide bandwidth with a centre frequency between 100-130 kHz. They were designed to operate effectively as both transmitters and receivers of ultrasound with a beam width of around 10 degrees at the device centre frequency. The maximum electrical conductance of the transducer in water is $1.14 \mathrm{~ms}$ at $92.6 \mathrm{kHz}$ and the $-3 \mathrm{~dB}$ bandwidth is $99 \mathrm{kHz}(72 \mathrm{kHz}$ to $171 \mathrm{kHz})$. The mechanical quality factor $(\mathrm{Q})$ is 1.23 (transmitter serial no 001, receiver serial no 003). The transmitter was driven directly with a $10 \mathrm{~V}$ peak-to-peak, 10-cycle tone burst from the function generator (see above). The receiver was connected directly into the oscilloscope. Both transducers were mounted in a small water tank at a separation of $0.5 \mathrm{~m}$. Signals were digitised using a Tektronix TDS 3034B oscilloscope. Measurements were made over a range of frequencies from 40 to $200 \mathrm{kHz}$. A total 
(a)

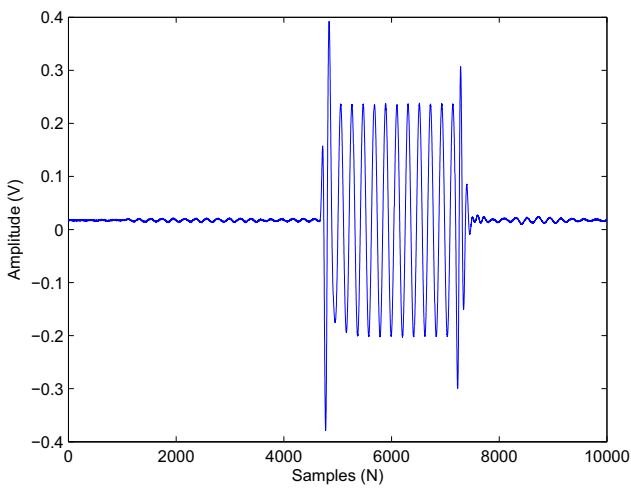

Fig1. Example of received signals: (a) $48 \mathrm{kHz}$. (b) $120 \mathrm{kHz}$

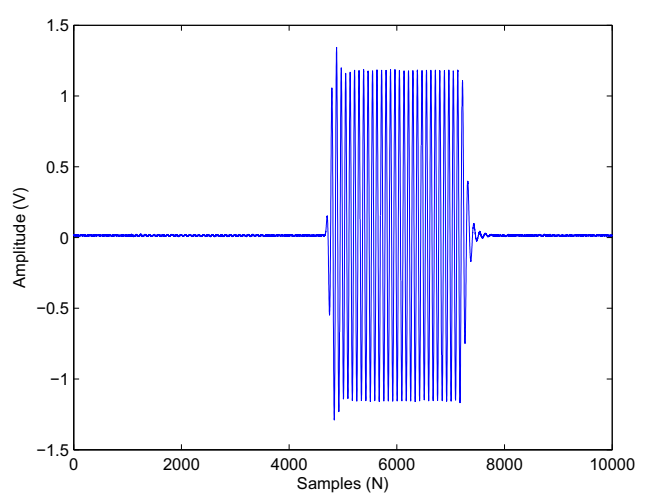

of 104 points were recorded for each waveform at each measurement frequency. This tone-burst approach is suited to ultrasonic transducers, which are specifically designed to be used at or close to their resonant frequency, allowing a good representation of the transducer's performance at discrete frequencies across its bandwidth. The transmitterreceiver separation of $0.5 \mathrm{~m}$ was selected to give a two-way travel time of $667 \mu \mathrm{s}$, where the sound velocity is $1500 \mathrm{~m} / \mathrm{s}$ in the surrounding medium (i.e. water). The sampling frequency was $10 \mathrm{MHz}$. This propagation time and sampling frequency, gave a signal length $\left(10^{7} \times 667 \times 10^{-6}\right)=6670$ samples, or in terms of frequency resolution (fs/N) $1500 \mathrm{~Hz}$. We decided to take just 2500 samples for each tone-burst to ensure $4 \mathrm{kHz}$ resolution. With this window length and frequency resolution, two sets of 41 signals each starting at $40 \mathrm{kHz}$, and rising in steps of $4 \mathrm{kHz}$, to $200 \mathrm{kHz}$ were generated. The 2500 samples used gave 10 cycles for the lowest frequency tone-burst $(40 \mathrm{kHz})$, which is enough to compute the Discrete Fourier Transform (DFT), for example. Using frequencies at this step interval enables the frequency to be determined exactly on a DFT frequency bin and hence, give an accurate measure and minimise spectrum leakage of the response at that frequency. Furthermore, with the length of signals chosen we also avoided interference from reflections from the tank walls. Both sine and cosine signals were generated at each of the calibration frequencies. These were designated r0.txt and $i 0 . t x t$ for the real (cosine) and imaginary (sine) signals for the first frequency set0, for example. The sets were sequentially numbered from 0 to 40 . An example of the received signals for the $48 \mathrm{kHz}$ and the $120 \mathrm{kHz}$ tone-burst signals is shown in figure1.

In order to provide a good estimation of the spectrum, we excluded samples affected by the switch on and switch off, of the resonant transducer (see figure 1), taking 700 samples either side of the centre of this 2500 samples long received 'toneburst' signal for our analyses. This provides 1400 samples about the centre of the 'tone burst' time window avoiding the effects of ringing and reflections. Consequently, we designed two sets of 41 test signals (0..40) each, real and imaginary, in steps of $4 \mathrm{kHz}$ starting at $40 \mathrm{kHz}$ and ending at $200 \mathrm{kHz}$. Each test signal was transmitted as a continuous sine and cosine wave 'tone-burst' having a $250 \mu \mathrm{s}$ duration. The discrete Fourier Transform (DFT) of the centre portion (1400 samples) of each received signal was performed. In fact, the only frequency computed and used for the calibration was the actual frequency of the test signal being analysed. Magnitude and phase responses were computed for all the 41 signals.

\section{Calibration}

Having obtained this set of 41-calibration values (via DFT of the sine and cosine sets) over the frequency range, we calculated the response (41 frequency bins) of the system in terms of magnitude and phase with respect to the frequency, as shown in figure 3. We can see from the figure 3 , there is a considerable variation at low and high frequencies in magnitude. Similarly, the phase in figure 6 is changing rapidly in the centre band of frequencies. Using these magnitude and phase responses, the signals were compensated then re-sent, by altering the amplitude and phase according to the 'toneburst' response functions in a such way as to create a flat response. The received signals were then used to obtain new sets of magnitude and phase responses as shown in figure 4 . Variations in the 
(a)

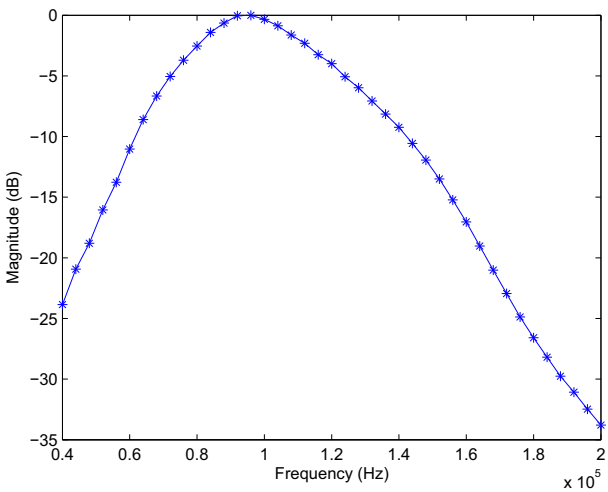

Fig2. Magnitude and phase responses: (b)

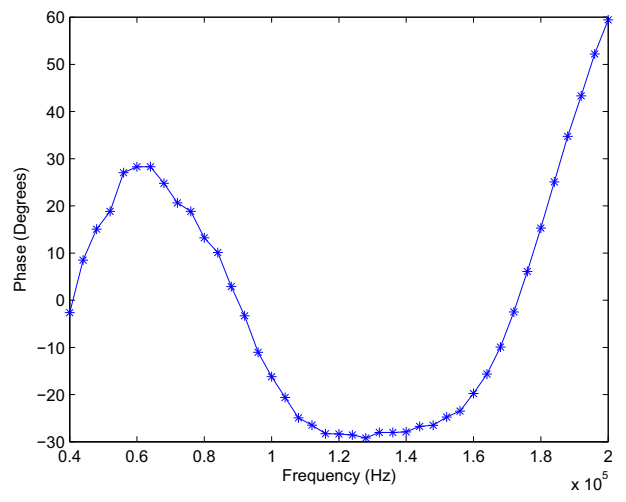

(a) Magnitude variation(35 dB). (b) Phase variation (90 degree) (a)

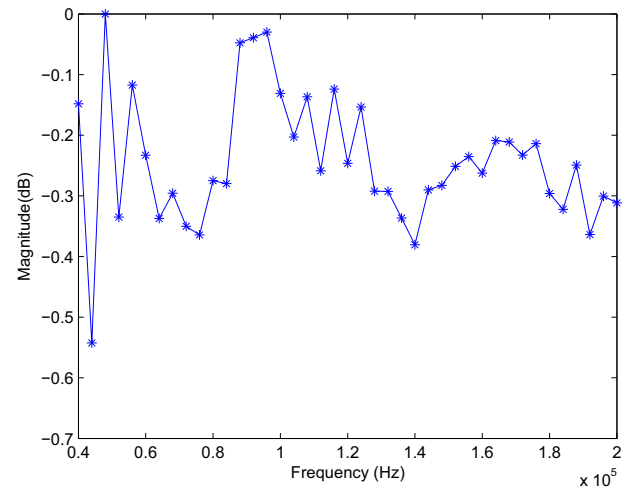

(b)

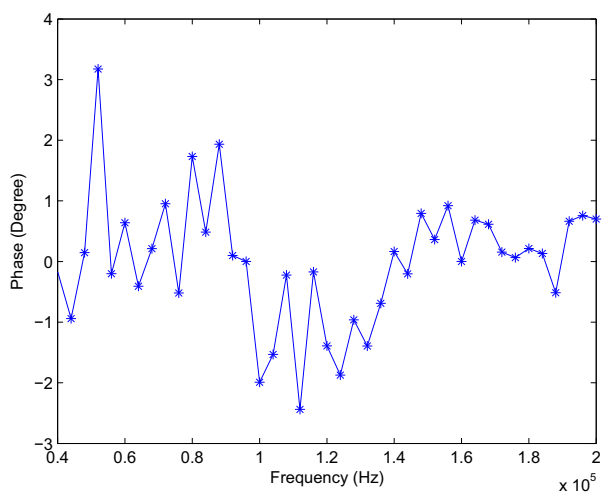

Fig3. Magnitude and phase responses after compensation: (a) $0.6 \mathrm{~dB}$ variation. (b) 6 degree variation

magnitude and phase responses can be seen to be drastically reduced, following our compensation (based on calibrating the whole system). The phase compensation is within \pm 3 degrees over the entire frequency band, a considerable improvement over the original 90-degrees variation. The magnitude was flat to within $1 \mathrm{~dB}$, again a considerably less than the original variation of $35 \mathrm{~dB}$ ( i.e. a 30 times reduction in the variation of both magnitude $(\mathrm{dB})$ and phase (degrees) responses).

\section{LINEAR CHIRP COMPENSATION}

In order to test the method, we applied it to compensate a chirp signal. Since we use responses at 41 discrete points in the frequency band, it was necessary to interpolate between discrete points to calculate the response at all the desired frequencies in order to compensate the chirp signal. We used the Matlab ${ }^{\circledR}$ 'interp1' function with 'cubic' interpolation. For the purposes of the calibration, 2500 points were used to generate the chirp signal at a sampling frequency of $10 \mathrm{MHz}$ (as described above). The 41 points of the magnitude and phase responses were interpolated to 2500 values using

$$
\text { new } A=\operatorname{interp} 1\left(t, T H R(2,:)^{\prime}, \text { newt },^{\prime} \text { cubic }{ }^{\prime}\right)
$$

where new $\mathrm{A}$ is the amplitude at the required new points, newt is the time of each of the 2500 new samples points, $\mathrm{t}$ is the time at the original 41 points and $\operatorname{THR}(2$, :) contains the original ' 41 value' magnitude response. A similar calculation was performed for phase using

$$
\text { newP }=\operatorname{interp} 1\left(t, \operatorname{THR}(3,:)^{\prime}, \text { newt },^{\prime} \text { cubic }{ }^{\prime}\right)
$$

where newP was an array of 2500 phase compensation values, and $\operatorname{THR}(3,:)$ contains the original '41values' phase response. Consequently, the compensation for both magnitude and phase could be achieved in one operation.

\section{Results AND discussion}

To validate the method, we selected a broadband chirp signal having a frequency range comparable to the transducer response. A Gaussian window was applied to the transmitted signal in order to minimise unwanted 'turn on', 'turn off' signals seen 
(a)

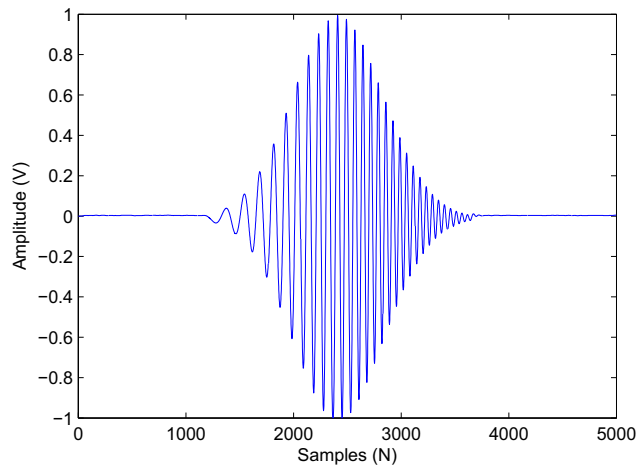

Fig4. Linear Gaussian chirp: (a) Original (Transmitted). (b) Compensated (transmitted)

(b)
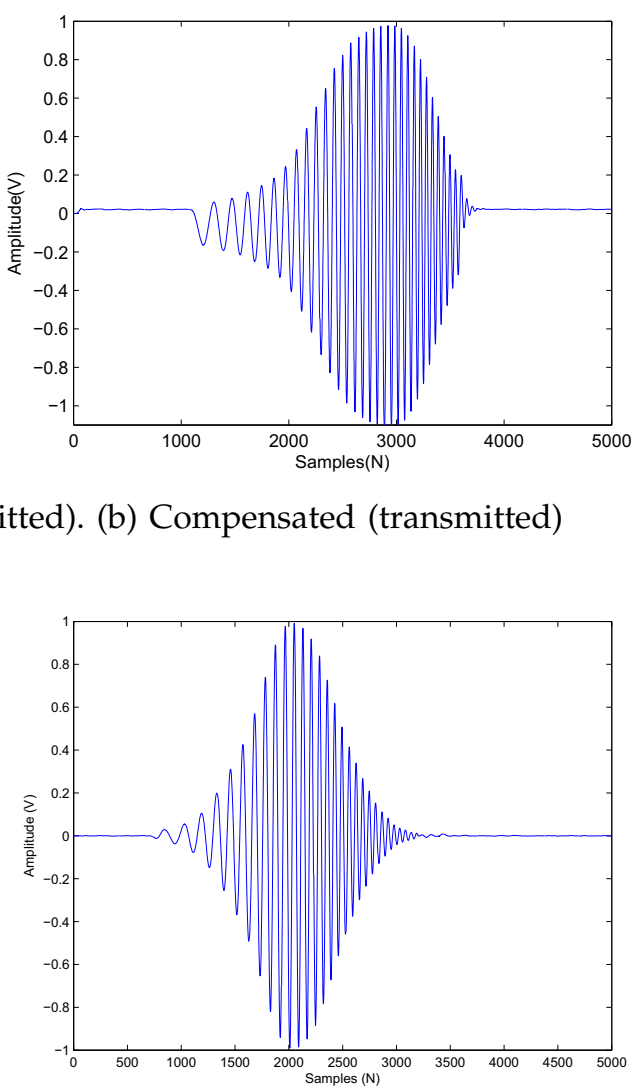

(b)

(a)

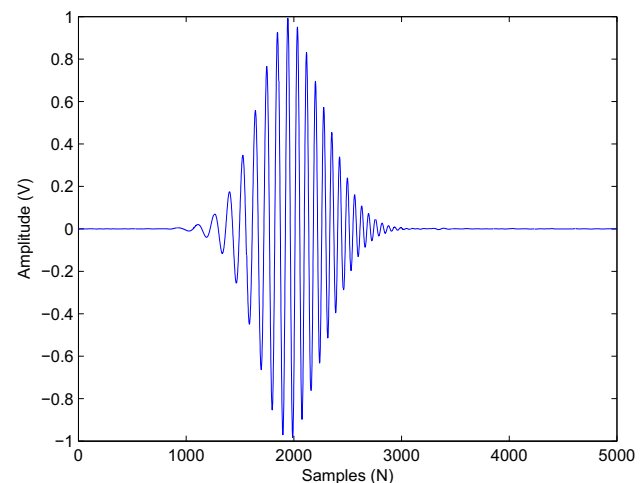

Fig5. Linear Gaussian chirp: (a) Original (received). (b) Compensated (received)

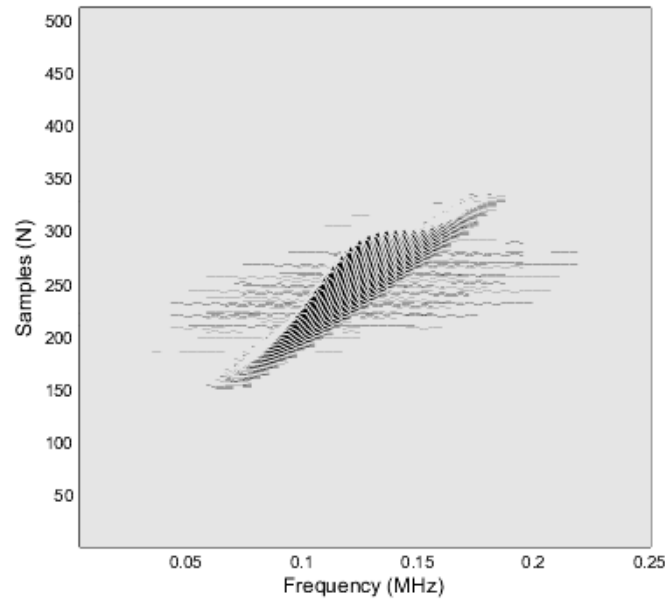

(a)

(b)

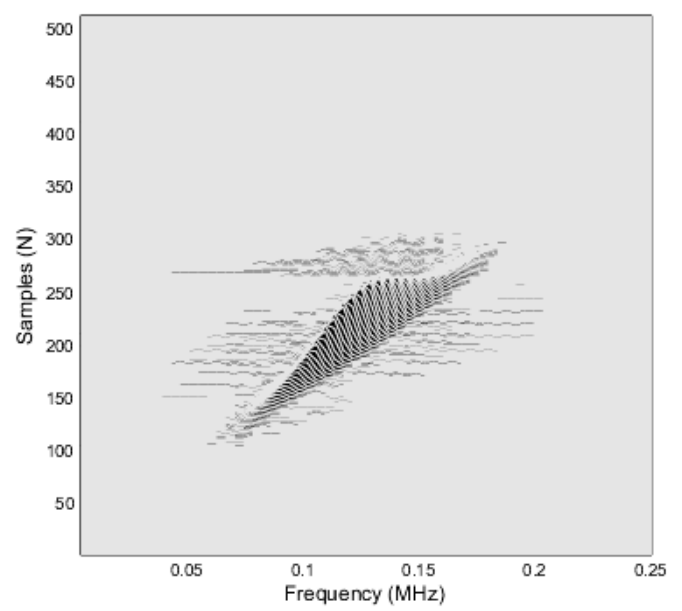

Fig6. Smoothed Wigner-Ville: (a) the transmitted original chirp. (b) the received compensated chirp originally (figure 1). The signals are shown in figure 5 after applying a digital low pass Butterworth filter $(0-400 \mathrm{kHz})$ to eliminate undesirable high frequencies. We can see a significantly different signal (figure $5 b$ ) to that propagated originally (figure $4 b$ ). The signal in figure $5 b$ is compensated in relation to the magnitude and phase response 'transferHR' developed as part of the software calibration. Figure $5 \mathrm{a}$ shows the signal received when the original Gaussian chirp (figure 4a) is transmitted. When the compensated signal was applied to the transmitter (figure $4 b$ ), a Gaussian chirp signal was received 
(figure 5b) similar to the original 'Gaussian' chirp (figure 4a). Thus, the compensation technique can be seen to be effective. A time-frequency analysis was undertaken using the original transmitted and compensated received chirp signal (after sub sampling the signals to $1 \mathrm{MHz}$ ) to show their resemblance. The results in figure $6 \mathrm{a}$ and $6 \mathrm{~b}$, using the smoothed Wigner-Ville distribution under the TFSA Matlab toolbox developed by Boashash [12], show the signals to be almost identical.

\section{CONCLUSION}

In this paper, we demonstrate a software method to calibrate whole ultrasonic transmitting-receiving systems for magnitude and particularly phase distortion. Distorting the input signal on the basis of the magnitude and phase response of the whole system enabled us to acquire the desired signal at the output with little distortion, using piezoelectric transducers in a broadband transmitting and receiving system. Using a linear chirp as a test signal, we validated this method over a range of frequencies, as the results showed close resemblance between the desired and received signals. Such system calibration is necessary when using ultrasonic techniques to characterise materials. For example, to control signal properties, otherwise the signals may not be sensitive to the analysis necessary to identify materials properties in terms of changes in their magnitude and phase. Such 'calibrated' signals are intended for use in experiments investigating techniques for improved imaging, physical properties characterisation of materials and investigation of material heterogeneity.

\section{ACKNOWLEDGMENTS}

This work was undertaken as part of the Biologically Inspired Acoustic Systems (BIAS) project that is funded by the RCUK via the Basic Technology Programme grant reference number EP/C523776/1. The BIAS project involves collaboration between the British Geological Survey, Leicester University, Fortkey Ltd., Southampton University, Leeds University, Edinburgh University and Strathclyde University.

The work of David Robertson and Victor Murray of Alba Ultrasound Ltd. in the design of the wideband piezo-composite transducers is gratefully acknowledged.

This paper is published with the permission of the Executive Director of the British Geological Survey (NERC).

\section{REFERENCES}

[1] Alexander Mofachern (1991) "Method and apparatus for calibrating transducer/amplifier systems," United States Patent, number 5014229.

[2] Hatano, Hajime and Mori, Eiji (1976) "Acoustic-emission transducer and its absolute calibration," Journal of the Acoustical Society of America, volume 59, number 2, pages 344-349.

[3] P.D. Theobald, T.J. Esward, S.P. Dowson, and R.C. Preston (2005) "Acoustic emission transducers-development of a facility for traceable out-of-plane displacement calibration". Ultrsaonics, volume 43, pages 343-350.

[4] T. Yan, P. Theobald and B.E. Jones (2004) "A canonical piezoelectric transducer with integral sensor as a selfcalibrating acoustic emission energy source," Ultrasonics, volume 42, pages 431-438.

[5] R.M. Fano (1950) "Theoretical Limitations of The Broadband Matching of Arbitrary Impedances". Franklin Institute, volume 244 , pages $57-83$,

[6] L.W. Schmerr, A. Lopez-Sanchez and R. Huang (2006) "Complete Ultrasonic transducer characterization and use for models and measurements," Utrasonics, volume 44, pages $753-757$.

[7] D. C. Youla (1964) "A new theory of broadband matching," IEEE Trans. Cir. Theory, volume 11, pages 30.

[8] T. M. Reeder, W.R. Schreve, and P.L. Adams (1972) "A New Broadband Coupling Network for Interdigital Surface wave Transducers," IEEE Trans. Sonics and Ultrasonics, volume 19, pages 466-469.

[9] J. Anderson and L. Wilkins (1979) "The design of optimum Lumped Broadband Equalizers for ultrasonics Transducers," J. Acous. Soc, volume 66, pages 629.

[10] P.E. Doust and J.F. Dix (2001) "The impact of improved transducer matching and equalisation techniques on the accuracy and validity of underwater acoustic measurements," Acoustical Oceanography', Proceeding of Institute of acoustics, Editor: T.G Leighton, G.J Heald, H. Griffiths and G. Griffiths, volume 23 Part2, pages 100-109.

[11] P.E Doust (2000) "Equalising Transfer Functions for Linear Electro-Acoustic Systems," UK Patent Application, number 0010820.9 .

[12] B. Boashash (2005) "Time-frequency signal analysis Toolbox: TFSA 5.41," Organization: Signal Processing Research Laboratory, ueensland University of Technology. 